\title{
Long-Term Experience of Sperm Cryopreservation in Cancer Patients in a Single Fertility Center
}

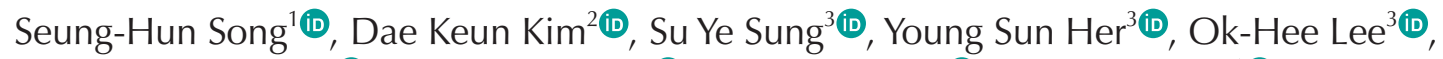 \\ Myoung Hwa Choi ${ }^{3}$, Hae Kyung Kim $^{3}$ (D), Sang Woo Lyu ${ }^{4}$, Dong Suk Kim ${ }^{1}$ (D) \\ ${ }^{1}$ Department of Urology, Fertility Center of CHA Gangnam Medical Center, CHA University College of Medicine, ${ }^{2}$ Department of Urology, \\ CHA Fertility Center, Seoul Station, CHA University College of Medicine, ${ }^{3}$ Fertility Center of CHA Gangnam Medical Center, CHA \\ University College of Medicine, ${ }^{4}$ Department of Obstetrics and Gynecology, Fertility Center of CHA Gangnam Medical Center, CHA \\ University College of Medicine, Seoul, Korea
}

\begin{abstract}
Purpose: Sperm cryopreservation before cancer treatment is the most effective method to preserve the fertility of male patients. We present our 21 years experience with sperm cryopreservation for cancer patients, including an examination of semen quality, the current status of cryopreserved sperm, and the rate of sperm use for assisted reproductive technology (ART). Materials and Methods: A total of 721 cancer patients at Fertility Center of CHA Gangnam Medical Center successfully performed sperm cryopreservation for fertility preservation from January 1996 to December 2016. Medical chart review was used to analyze patient age, marital status, cancer type, semen volume, sperm counts and motility, length of storage, and current banking status.

Results: The major cancers of the 721 patients were leukemia (28.4\%), lymphoma (18.3\%), testis cancer (10.0\%). The mean age at cryopreservation was 27.0 years, and 111 patients (15.4\%) performed sperm cryopreservation during or after cancer treatment. The mean sperm concentration was $66.7 \pm 66.3 \times 10^{6} / \mathrm{mL}$ and the mean sperm motility was $33.8 \% \pm 16.3 \%$. During median follow-up duration of 75 months (range, 1-226 months), 44 patients $(6.1 \%)$ used their banked sperm at our fertility center for ART and 9 patients (1.2\%) transferred their banked sperm to another center. The median duration from cryopreservation to use was 51 months (range, 1-158 months).

Conclusions: Sperm cryopreservation before gonadotoxic treatment is the most reliable method to preserve the fertility of male cancer patients. Sperm cryopreservation should be offered as a standard of care for all men planning cancer therapy.
\end{abstract}

Keywords: Cryopreservation; Fertility; Neoplasms; Semen preservation

This is an Open Access article distributed under the terms of the Creative Commons Attribution Non-Commercial License (http://creativecommons.org/licenses/by-nc/4.0) which permits unrestricted non-commercial use, distribution, and reproduction in any medium, provided the original work is properly cited.

\section{INTRODUCTION}

In 2014, there were 112,882 newly diagnosed cases of cancer among males in South Korea [1]. The development of new chemotherapy and radiotherapy techniques has increased the survival rate for many types of cancer from $50 \%$ in the 1970 s to $70 \%$ in 1990 s [2]. The current cure rate for patients with testicular cancer and lymphoma are as high as $90 \%$ [2]. However, cancer therapies, including chemotherapy and radiation therapy, can impair male reproductive function by directly damaging the germinal epithelium, causing ol-

Received: Jul 13, 2018 Revised: Nov 19, 2018 Accepted: Dec 5, 2018 Published online Dec 26, 2018

Correspondence to: Dong Suk Kim iD https://orcid.org/0000-0001-7350-0303

Department of Urology, Fertility Center of CHA Gangnam Medical Center, 566 Nonhyeon-ro, Gangnam-gu, Seoul 06135, Korea.

Tel: +82-2-3468-3413, Fax: +82-2-3468-3449, E-mail: dngskkim100@gmail.com 
igozoospermia or azoospermia, or by damaging the neural pathways that regulate erection and ejaculation [3]. In addition, cytotoxic chemotherapy may also lead to transmission of genetic damage to children conceived after tretment [3]. The effect of cancer treatment on the incidence and severity of testicular dysfunction depends on various factors, including type of therapeutic regimen and cumulative dose [4].

It is difficult to predict which cancer patients will recover spermatogenesis function and which will remain infertile. Therefore sperm cryopreservation before cancer treatment is the most valuable and effective method to preserve the reproductive capacity for males of reproductive age undergoing cancer treatment [5]. Moreover, a patient's knowledge that his fertility can be secured through sperm cryopreservation may help in the emotional battle against cancer [6].

Fertility Center of CHA Gangnam Medical Center (Seoul, Korea) has performed sperm cryopreservation in patients diagnosed with cancer since January 1996. We evaluated data from our 21 years of experience with banked sperm by examination of cancer type, semen quality at banking, and current status of cryopreserved sperm including use rate or ongoing preservation rate. These long-term data could be helpful for oncologists and patients who are considering sperm cryopreservation before cancer treatment.

\section{MATERIALS AND METHODS}

\section{Subject and study design}

A total of 721 patients with cancer successfully performed sperm cryopreservation for fertility preservation through masturbation at our fertility center from January 1996 to December 2016. Patients who failed to cryopreserve semen because of azoospermia or having insufficient sperm quality for freezing, or who banked testicular sperm after testicular sperm extraction were not included. The medical charts of all patients were retrospectively reviewed and data on patient age, marital status, cancer type (determined by the oncologist's referral document), semen volume, sperm count and motility, length of storage, and current banking status were extracted. All semen samples were collected in sterile plastic containers by masturbation. After liquefaction, semen parameters were determined according to World Health Organization guidelines at the time of sample collection [7-9]. Semen samples were cryopre- served if motile sperm were present. Semen was mixed with the same volume of Sperm Freezing Medium (Origio, Denmark), and then incubated at room temperature for 10 minutes. The mixture was then dispensed into cryogenic tubes consisting of ionomer resin $\left(\mathrm{CBS}^{\mathrm{TM}}\right.$ High Security cotton-plugged sperm straws; Cryo Bio System, L'Aigle, France). Prepared samples were suspended just above the surface of liquid nitrogen for 30 minutes, and then immersed into the liquid nitrogen $\left(-196^{\circ} \mathrm{C}\right)$.

The initial duration of sperm cryopreservation was 3 years. Then, freezing was extended each subsequent year after confirmation by direct contact or a telephone call with the patient or legal guardian. Patients had the option to discard the frozen samples or to proceed with assisted reproductive technology (ART) at another fertility center, in which case the sample was transferred. If the patient died during or after cancer treatment, all samples were discarded. The current banking status (determined in October 2017) includes samples used in our fertility center, transferred to other centers, electively discarded, discarded secondary to death, and those still in storage.

\section{Ethics statement}

The study protocol was reviewed and approved by the Institutional Review Board of CHA Gangnam Medical Center of CHA University (Reg. No. GCI-17-83).

All patients provided informed written consent before sperm cryopreservation. If the patient was a minor, informed written consent was obtained from the parents or legal guardians. For married patients, spousal consent was required for sperm banking.

\section{Statistical analysis}

All data are presented as means \pm standard deviations or medians and ranges. The significance of differences between groups was evaluated using analysis of variance (ANOVA) and the Mann-Whitney U-test. An alpha value of $5 \%$ was considered the threshold for significance. SPSS ver. 12.0K (SPSS Inc., Chicago, I, USA) was used for statistical analysis.

\section{RESULTS}

The major types of cancer in the 721 patients (Table 1) were leukemia $(n=205,28.4 \%)$, lymphoma $(n=132,18.3 \%)$, testis cancer $(n=72,10.0 \%)$, severe aplastic anemia $(n=61$, 
8.5\%), sarcoma ( $\mathrm{n}=35,4.8 \%)$, thyroid cancer $(\mathrm{n}=29,4.0 \%)$, rectal cancer $(n=20,2.8 \%)$, myelodysplastic syndrome $(\mathrm{n}=18,2.5 \%)$, brain tumor $(\mathrm{n}=10,1.4 \%)$, stomach cancer

Table 1. Cancer type of patients who underwent sperm cryopreservation

\begin{tabular}{lc}
\hline \multicolumn{1}{c}{ Cancer type } & No. of patient (\%) \\
\hline Leukemia & $205(28.4)$ \\
Lymphoma & $132(18.3)$ \\
Testis cancer & $72(10.0)$ \\
Severe aplastic anemia & $61(8.5)$ \\
Sarcoma & $35(4.8)$ \\
Thyroid cancer & $29(4.0)$ \\
Rectal cancer & $20(2.8)$ \\
Myelodysplastic syndrome & $18(2.5)$ \\
Brain tumor & $10(1.4)$ \\
Stomach cancer & $9(1.2)$ \\
Lung cancer & $7(1.0)$ \\
Other hematologic cancer & $7(1.0)$ \\
Colon cancer & $6(0.8)$ \\
Prostate cancer & $5(0.7)$ \\
Mediastinal cancer & $5(0.7)$ \\
Multiple myeloma & $5(0.7)$ \\
Other cancer & $36(5.0)$ \\
Unknown & $59(8.2)$ \\
Total & $721(100)$ \\
\hline
\end{tabular}

$(n=9,1.2 \%)$, lung cancer $(n=7,1.0 \%)$, other hematologic cancers $(n=7,1.0 \%)$, colon cancer $(n=6,0.8 \%)$, prostate cancer $(n=5,0.7 \%)$, mediastinal cancer $(n=5,0.7 \%)$, and multiple myeloma $(\mathrm{n}=5,0.7 \%)$. There were fewer than 5 patients per cancer type for 36 patients $(5.0 \%$; including bladder cancer, skin cancer, retroperitoneal tumor, breast cancer, urachal cancer, biliary duct cancer, and tongue cancer) and 59 patients (8.2\%) had insufficient medical chart information. Analysis of marital status indicated 584 patients (81.0\%) were unmarried, 80 (11.1\%) were married, and 57 (7.9\%) had unknown status.

The mean patient age at sperm cryopreservation was 27.0 7.9 years (range, 13-65 years). A total of 1,120 sperm samples were collected, and there was a mean of $3.1 \pm 1.7$ samples per patient (range, $1-10$ ). The mean sperm concentration was $66.7 \pm 66.3 \times 10^{6} / \mathrm{mL}$ (range, $0.09-420 \times 10^{6} / \mathrm{mL}$ ) and the mean sperm motility was $33.8 \% \pm 16.3 \%$ (range, $0 \%-77 \%$ ).

A one-way ANOVA indicated a relationship of semen parameters with type of cancer (Table 2). In particular, the sperm concentration in patients with testis cancer $\left(37.9 \pm 40.5 \times 10^{6} / \mathrm{mL}\right)$ was significantly lower than that of patients with other cancers, including leukemia, lymphoma, sarcoma, thyroid cancer, rectal cancer,

Table 2. Semen characteristics at cryopreservation according to cancer type

\begin{tabular}{|c|c|c|c|c|c|}
\hline Cancer type & Age (y) & $\begin{array}{c}\text { Semen } \\
\text { volume }(\mathrm{mL})\end{array}$ & $\begin{array}{c}\text { No. of } \\
\text { frozen vial }\end{array}$ & $\begin{array}{c}\text { Sperm } \\
\text { concentration } \\
\left(10^{6} / \mathrm{mL}\right)\end{array}$ & $\begin{array}{c}\text { Sperm } \\
\text { motility (\%) }\end{array}$ \\
\hline Leukemia & $24.4 \pm 6.0$ & $3.1 \pm 1.7$ & $2.7 \pm 1.6$ & $66.3 \pm 79.4$ & $28.1 \pm 16.6$ \\
\hline Lymphoma & $26.6 \pm 7.1$ & $3.0 \pm 1.6$ & $3.4 \pm 1.6$ & $88.2 \pm 63.8$ & $38.2 \pm 15.5$ \\
\hline Testis cancer & $26.8 \pm 6.5$ & $3.1 \pm 1.6$ & $3.1 \pm 1.8$ & $37.9 \pm 40.5$ & $35.2 \pm 13.8$ \\
\hline Severe aplastic anemia & $25.4 \pm 6.8$ & $3.4 \pm 2.1$ & $3.1 \pm 1.6$ & $54.4 \pm 52.9$ & $32.6 \pm 15.2$ \\
\hline Sarcoma & $22.1 \pm 7.1$ & $2.6 \pm 1.8$ & $2.9 \pm 1.3$ & $77.2 \pm 90.5$ & $36.8 \pm 13.0$ \\
\hline Thyroid cancer & $35.0 \pm 7.0$ & $3.2 \pm 1.7$ & $3.4 \pm 1.2$ & $85.0 \pm 52.3$ & $42.6 \pm 12.6$ \\
\hline Rectal cancer & $35.5 \pm 7.4$ & $2.1 \pm 0.9$ & $2.9 \pm 1.3$ & $90.7 \pm 51.2$ & $40.9 \pm 12.7$ \\
\hline Myelodysplastic syndrome & $29.1 \pm 5.9$ & $3.2 \pm 1.8$ & $3.7 \pm 1.9$ & $66.7 \pm 45.7$ & $34.8 \pm 14.6$ \\
\hline Brain tumor & $24.9 \pm 7.1$ & $3.3 \pm 1.9$ & $3.0 \pm 1.8$ & $55.3 \pm 35.4$ & $33.4 \pm 11.2$ \\
\hline Stomach cancer & $37.1 \pm 5.7$ & $2.6 \pm 0.6$ & $3.2 \pm 1.0$ & $87.6 \pm 66.3$ & $38.3 \pm 10.4$ \\
\hline Lung cancer & $31.9 \pm 5.9$ & $3.0 \pm 1.0$ & $3.3 \pm 1.0$ & $48.7 \pm 21.9$ & $34.3 \pm 19.3$ \\
\hline Other hematologic cancer & $23.0 \pm 5.9$ & $4.1 \pm 2.0$ & $4.3 \pm 1.5$ & $73.0 \pm 60.5$ & $44.6 \pm 16.6$ \\
\hline Colon cancer & $41.3 \pm 6.7$ & $2.8 \pm 1.4$ & $3.2 \pm 1.3$ & $88.6 \pm 49.3$ & $37.8 \pm 19.6$ \\
\hline Prostate cancer & $54.8 \pm 6.5$ & $1.0 \pm 0.9$ & $2.2 \pm 1.3$ & $66.2 \pm 62.9$ & $23.8 \pm 19.8$ \\
\hline Mediastinal cancer & $22.6 \pm 4.6$ & $3.4 \pm 1.7$ & $3.4 \pm 1.5$ & $54.0 \pm 39.7$ & $37.2 \pm 10.9$ \\
\hline Multiple myeloma & $34.8 \pm 5.6$ & $4.9 \pm 2.8$ & $4.4 \pm 0.5$ & $66.4 \pm 22.3$ & $29.6 \pm 12.8$ \\
\hline Total & $27.0 \pm 7.9$ & $3.0 \pm 1.7$ & $3.1 \pm 1.7$ & $66.7 \pm 66.3$ & $33.8 \pm 16.3$ \\
\hline $\mathrm{p}$-value & $<0.001$ & 0.017 & 0.014 & 0.002 & $<0.001$ \\
\hline
\end{tabular}

Values are presented as mean \pm standard deviation. 
Table 3. Semen characteristics at cryopreservation according to cancer treatment

\begin{tabular}{lccc}
\hline \multicolumn{1}{c}{ Variable } & $\begin{array}{c}\text { Sperm banking without } \\
\text { cancer treatment }\end{array}$ & $\begin{array}{c}\text { Sperm banking during or } \\
\text { after cancer treatment }\end{array}$ & p-value \\
\hline Patients & $610(84.6)$ & $111(15.4)$ & 0.56 \\
Semen volume $(\mathrm{mL})$ & $3.0 \pm 1.7$ & $3.1 \pm 1.8$ & $<0.001$ \\
Sperm concentration $\left(10^{6} / \mathrm{mL}\right)$ & $71.9 \pm 67.2$ & $33.6 \pm 48.7$ & $<0.001$ \\
Sperm motility $(\%)$ & $35.2 \pm 15.6$ & $24.2 \pm 17.3$ & \\
\hline
\end{tabular}

Values are presented as number (\%) or mean \pm standard deviation.

and myelodysplastic syndrome. This may be because 40 of the 72 patients (55.6\%) with testis cancer received unilateral radical orchiectomy before sperm cryopreservation. Sperm motility in patients with leukemia $(28.1 \% \pm 16.6 \%)$ was significantly lower than that of patients with lymphoma, testis cancer, sarcoma, thyroid cancer, rectal cancer, and stomach cancer (Table 2).

In spite of recommendations to perform sperm banking before cancer treatment, 111 patients (15.4\%) underwent sperm cryopreservation during or after cancer treatment. Relative to patients who banked sperm before cancer treatment, those who banked sperm during or after cancer treatment had a significantly lower sperm concentration $(\mathrm{p}<0.001)$ and lower sperm motility $(\mathrm{p}<0.001)$ (Table 3).

Our fertility center initially banks the sperm of cancer patients for 3 years, and provides additional storage for 1 year at a time after patient confirmation. During the follow-up (median duration, 75 months; range, 1-226 months), 44 patients (6.1\%) used banked sperm from our fertility center for ART, 9 patients (1.2\%) transferred their banked sperm to another center before starting ART, samples from 32 patients (4.4\%) were discarded due to patient death, 262 patients (36.3\%) electively discarded their samples, and 374 patients (51.9\%) requested continuation of storage (Fig. 1). Among the 44 patients who used banked sperm for ART, the median duration from sperm cryopreservation to sperm use was 51 months (range, 1-158 months). Analysis of the types of cancer in these 44 patients indicated that 9 had thyroid cancer, 7 had lymphoma, 6 had leukemia, 5 had severe aplastic anemia, 4 had sarcoma, 1 had testis cancer, 1 had rectal cancer, 1 had prostate cancer, 1 had urachal cancer, 1 had bile duct cancer, and 8 had unknown types of cancer. Thus, the overall rate of sperm use for ART was 31.0\% (9/29) for thyroid cancer patients, $5.3 \%$ (7/132) for lymphoma patients, $2.9 \%$ (6/205) for leukemia patients, $8.2 \%$ (5/61) for severe aplastic anemia patients, and $11.4 \%$ (4/35) for sarcoma patients.

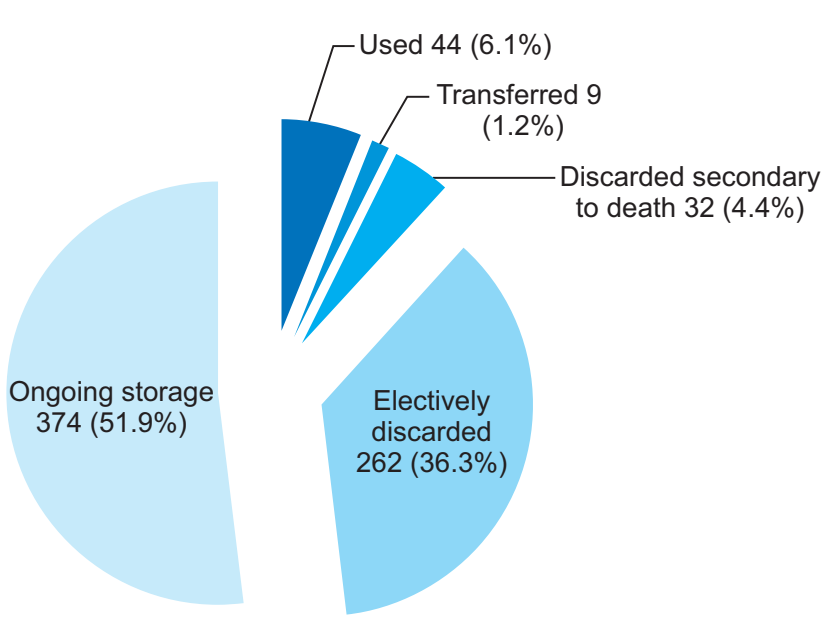

Fig. 1. Current status of cryopreserved sperm in 721 patients with malignancy (at October 2017).

However, because of the small number of patients who used their sperm for ART during the study period, longer investigations are needed to confirm any effect of cancer type on use of ART. Among the 44 patients who used banked sperm for ART, ART information was only available for only 38 patients. Clinical pregnancy was confirmed in 22 patients by ultrasound visualization of a gestational sac. Sperm used for ART after 158 months of cryopreservation led to clinical pregnancy in 2 patients.

\section{DISCUSSION}

The use of chemotherapy or radiotherapy to treat various cancers in men may lead to temporary, longterm, or permanent gonadal toxicities, depending on the type and dose of the therapeutic agent [10]. More specifically, chemotherapy induces the depletion or arrest of spermatogonial differentiation and mutagenesis in cells at later stages of development [4]. In contrast, radiotherapy affects the spermatogonia, the most radiosensitive cells due to their intense mitotic activity and spermatids [4]. Spermatids are unprotected because 
they lack mechanisms for DNA repair due to postmeiotic differentiation and chromatin condensation [4,11]. Patients receiving high doses of alkylating agents are very likely to become azoospermic, although spermatogenesis may recover over time [12]. Although male cancer patients receiving low doses of cytostatic agents may expect recovery of spermatogenesis at about 12 weeks after the end of chemotherapy, permanent azoospermia occurs in more than $50 \%$ of patients receiving high doses of these drugs [13]. All men with cancer who are of reproductive age should be counseled regarding the possible effects of cancer treatment on testicular function and risk of potential sperm DNA damage. Previous research indicated an increased frequency of sperm aneuploidy after the initiation of chemotherapy, and that this may persist up to 18 months or longer [14]. The American Society of Reproductive Medicine Ethics Committee and the American Society of Clinical Oncology both recommended that physicians inform all cancer patients about options for fertility preservation before treatment $[15,16]$. If this is not possible, then patients should be warned of the increased potential of genetic damage to sperm after initiation of chemotherapy or radiotherapy [3]. Although sperm banking is recommended before cancer treatment to obtain good sperm quality and to prevent potential DNA damage, some patients undergo cryopreservation during or after cancer treatment because of the urgency of treatment or a lack of information on the best methods for preservation of fertility. A survey by Schover et al [17] showed that although $91 \%$ of USA oncologists agreed that sperm banking should be offered to all male cancer patients, $48 \%$ admitted to never bringing up the topic or mentioning it to less than $25 \%$ of eligible men. The present study of 721 men with cancer indicated that 111 patients (15.4\%) performed sperm cryopreservation during or after cancer treatment. As expected, patients who banked sperm during or after cancer treatment had significantly lower sperm concentrations and lower sperm motility than those who banked their sperm before cancer treatment. This supports the policy that sperm banking should be offered to all men undergoing potentially gonadotoxic cancer therapy before the onset of treatment. Regular communication between oncologists and reproductive specialists and provision of complete information to patients of reproductive age is required not to miss the chance or not to be too late for fertility preservation.
Previous reports on sperm banking in cancer patients demonstrated that testicular cancer patients had the lowest median pre-thaw counts and motility [18,19]. $\mathrm{Ku}$ et al [20] performed a small retrospective study on sperm banking and reported that patients with testis cancer had significantly lower sperm concentrations than those with other cancers. van Casteren et al [21] noted that poor semen quality was common before the start of chemotherapy in men with testicular tumors, especially those with a non-seminoma testis cancer. In addition, Williams et al [22] showed that men with pretreatment testicular cancer had significantly lower semen quality than those with other malignancies, and suggested the possible causes were preexisting defects in germ cells, local tumor effects, endocrine disturbances, and autoimmune and systemic effects of cancer [23]. We found that the sperm concentration of patients with testis cancer $\left(37.9 \pm 40.5 \times 10^{6} / \mathrm{mL}\right)$ was significantly lower than that of patients with other cancers including leukemia, lymphoma, sarcoma, thyroid cancer, rectal cancer, and myelodysplastic syndrome. This may be because $40 / 72$ patients (55.6\%) with testis cancer received unilateral radical orchiectomy before sperm cryopreservation.

Among our 721 cancer patients who performed sperm cryopreservation, 44 (6.1\%) used their banked sperm at our fertility center for ART and 9 (1.2\%) transferred their banked sperm to another center for ART during median follow-up duration of 75 months (range, 1 to 226 months). The median duration from cryopreservation to sperm use for ART was 51 months (range, 1 to 158 months), which is similar to previous report. van Casteren et al [24] reported that among 557 patients who banked semen, 42 (7.5\%) requested use of the banked sperm after a mean time of 57 months (range, 15 to 130 months). If we classify transferred sperm as being used for ART, 7.4\% of our banked sperm was used for ART. The rate of banked sperm use in our study is comparable with that reported in other large studies. The rate of cryopreserved semen use among cancer patients is often less than $10 \%$ to $15 \%$, and differs widely among studies [24]. A recent systemic review showed the rate of cryopreserved semen use among cancer patients ranged between $2 \%$ and $60 \%$, with an aggregate value of $8 \%$ (95\% confidence interval, $8 \%-9 \%$ ) [25]. Patient survival, recovery of spermatogenesis, and followup duration could all affect the rate of banked sperm use. We expect the use of banked sperm will increase 
over time, as more men decide to use their semen to conceive children. Therefore, longer follow-up data are needed to estimate the cumulative rate of frozen sperm use.

The limitations of our study include its retrospective design and the lack of detailed data on ART outcomes including live birth rate. However, to our knowledge, this study is among the largest studies and used among the longest follow-up periods to examine cryopreserved semen of cancer patients in a single fertility center. These long-term data could be helpful for oncologists and patients who are considering sperm cryopreservation before cancer treatment.

\section{CONCLUSIONS}

Sperm cryopreservation before gonadotoxic treatment is the most reliable and noninvasive method to preserve male fertility. Sperm cryopreservation should be offered to all men who plan to receive cancer therapy. Efficient communication and counseling among the oncologist, patient, and reproductive specialist is mandatory so that fertility can be preserved for cancer patients of reproductive age. Even though only $6.1 \%$ of our patients used their banked sperm at our fertility center for ART and 1.2\% transferred their sperm to another center for ART, we expect that a large number of men will use their samples for ART in the future.

\section{ACKNOWLEDGEMENTS}

This work was supported by the National Research Foundation of Korea (NRF) grant funded by the Korea government (MSIT) (No. 2018R1C1B5083794).

\section{Disclosure}

The authors have no potential conflicts of interest to disclose.

\section{Author Contribution}

Design of study: Song SH, Kim DS. Data collection: Sung SY, Her YS, Lee OH, Choi MH, Kim HK. Analysis of data: Kim DK, Lyu SW, Kim DS. Review and correction: Song SH, Kim DS. All authors have read and approved the final manuscript.

\section{REFERENCES}

1. Jung KW, Won YJ, Oh CM, Kong HJ, Lee DH, Lee KH; Community of Population-Based Regional Cancer Registries. Cancer statistics in Korea: incidence, mortality, survival, and prevalence in 2014. Cancer Res Treat 2017;49:292-305.

2. Siegel R, DeSantis C, Virgo K, Stein K, Mariotto A, Smith T, et al. Cancer treatment and survivorship statistics, 2012. CA Cancer J Clin 2012;62:220-41.

3. Loren AW, Mangu PB, Beck LN, Brennan L, Magdalinski AJ, Partridge $\mathrm{AH}$, et al. Fertility preservation for patients with cancer: American Society of Clinical Oncology clinical practice guideline update. J Clin Oncol 2013;31:2500-10.

4. Trottmann M, Becker AJ, Stadler T, Straub J, Soljanik I, Schlenker B, et al. Semen quality in men with malignant diseases before and after therapy and the role of cryopreservation. Eur Urol 2007;52:355-67.

5. Tomlinson M, Meadows J, Kohut T, Haoula Z, Naeem A, Pooley K, et al. Review and follow-up of patients using a regional sperm cryopreservation service: ensuring that resources are targeted to those patients most in need. Andrology 2015;3:709-16

6. Saito K, Suzuki K, Iwasaki A, Yumura Y, Kubota Y. Sperm cryopreservation before cancer chemotherapy helps in the emotional battle against cancer. Cancer 2005;104:521-4.

7. World Health Organization. WHO laboratory manual for the examination of human semen and semen-cervical mucus interaction. 3rd ed. Cambridge: Cambridge University Press; 1992.

8. World Health Organization. WHO laboratory manual for the examination of human semen and sperm-cervical mucus interaction. 4th ed. Cambridge: Cambridge University Press; 1999.

9. World Health Organization. WHO laboratory manual for the examination of human semen. 5th ed. Geneva: World Health Organization; 2010.

10. Meistrich ML. Effects of chemotherapy and radiotherapy on spermatogenesis in humans. Fertil Steril 2013;100:1180-6.

11. Gandini L, Sgrò P, Lombardo F, Paoli D, Culasso F, Toselli L, et al. Effect of chemo-or radiotherapy on sperm parameters of testicular cancer patients. Hum Reprod 2006;21:2882-9.

12. van der Kaaij MA, van Echten-Arends J, Simons AH, KluinNelemans HC. Fertility preservation after chemotherapy for Hodgkin lymphoma. Hematol Oncol 2010;28:168-79.

13. Pont J, Albrecht W. Fertility after chemotherapy for testicular germ cell cancer. Fertil Steril 1997;68:1-5.

14. De Mas P, Daudin M, Vincent MC, Bourrouillou G, Calvas P, Mieusset $\mathrm{R}$, et al. Increased aneuploidy in spermatozoa from 
testicular tumour patients after chemotherapy with cisplatin, etoposide and bleomycin. Hum Reprod 2001;16:1204-8.

15. Ethics Committee of the American Society for Reproductive Medicine. Fertility preservation and reproduction in cancer patients. Fertil Steril 2005;83:1622-8.

16. Lee SJ, Schover LR, Partridge AH, Patrizio P, Wallace WH, Hagerty K, et al. American Society of Clinical Oncology recommendations on fertility preservation in cancer patients. J Clin Oncol 2006;24:2917-31.

17. Schover LR, Brey K, Lichtin A, Lipshultz LI, Jeha S. Oncologists' attitudes and practices regarding banking sperm before cancer treatment. J Clin Oncol 2002;20:1890-7.

18. Menon S, Rives N, Mousset-Siméon N, Sibert L, Vannier JP, Mazurier S, et al. Fertility preservation in adolescent males: experience over 22 years at Rouen University Hospital. Hum Reprod 2009;24:37-44.

19. Crha I, Ventruba P, Zakova J, Huser M, Kubesova B, Hudecek $\mathrm{R}$, et al. Survival and infertility treatment in male cancer patients after sperm banking. Fertil Steril 2009;91:2344-8.

20. Ku JY, Park NC, Jeon TG, Park HJ. Semen analysis in cancer patients referred for sperm cryopreservation before chemotherapy over a 15-year period in Korea. World J Mens Health 2015;33:8-13.

21. van Casteren NJ, Boellaard WP, Romijn JC, Dohle GR. Gonadal dysfunction in male cancer patients before cytotoxic treatment. Int J Androl 2010;33:73-9.

22. Williams DH 4th, Karpman E, Sander JC, Spiess PE, Pisters LL, Lipshultz LI. Pretreatment semen parameters in men with cancer. J Urol 2009;181:736-40.

23. Agarwal A, Allamaneni SS. Disruption of spermatogenesis by the cancer disease process. J Natl Cancer Inst Monogr 2005:912.

24. van Casteren NJ, van Santbrink EJ, van Inzen W, Romijn JC, Dohle GR. Use rate and assisted reproduction technologies outcome of cryopreserved semen from 629 cancer patients. Fertil Steril 2008;90:2245-50.

25. Ferrari S, Paffoni A, Filippi F, Busnelli A, Vegetti W, Somigliana E. Sperm cryopreservation and reproductive outcome in male cancer patients: a systematic review. Reprod Biomed Online 2016;33:29-38. 